\title{
Zweifel und Verzweiflung
}

Andreas Hunziker

1.

Annemarie Piepers Artikel `Verzweiflung im Lexikon Existenzialismus und Existenzphilosophie beginnt folgendermassen:

"Verzweiflung als existenzielle Befindlichkeit ist radikalisierter Selbstzweifel, der den ganzen Menschen, nicht nur den Kopf erfasst. Wer an sich verzweifelt, ist außerstande, sich als seiner selbst mächtiges, autonomes Individuum zu verstehen und in einem geglückten Selbstverhältnis zur Einheit mit sich selbst zu gelangen. Anstatt den seine eigene Existenz konstituierenden Gegensatz von Körper und Geist zu überwinden, schreibt es die von ihm als innere Zerrissenheit erlebte und erlittene Dualität seines Seins in der Hoffnungslosigkeit fest." (Art. >Verzweiflung〈, 280)

Kierkegaards Begriff derVerzweiflung, wie wir ihn aus der Krankheit zum Tode kennen, ist in diesen Sätzen mit Händen zu greifen. Pieper fährt denn auch weiter, indem sie referiert, wie Kierkegaard bereits in seiner frühen, Fragment gebliebenen Schrift Johannes Climacus oder De omnibus dubitandum est den Weg eines jungen Skeptikers beschreibt. Dieser junge Mensch mit dem Namen Johannes Climacus versucht, mit Descartes' Aufforderung, an allem zu zweifeln, radikal Ernst zu machen. Am Ende findet er aber nicht zur Selbstgewissheit des cogitans sum, sondern beginnt am Leben selbst zu verzweifeln. Dieser Umschlag vom theoretisch-epistemologischen Zweifel in den praktisch-existenziellen (Selbst-)Zweifel ist vorausgesetzt, wenn Anti-Climacus schliesslich in der relativ späten Schrift Die Krankheit zum Tode die bekannten drei Typen derVerzweiflung unterscheidet:

"Verzweiflung ist eine Krankheit im Geist, im Selbst, und kann somit ein Dreifaches sein: verzweifelt sich nicht bewußt sein, ein Selbst zu haben (uneigentliche Verzweiflung); verzweifelt nicht man selbst sein wollen; verzweifelt man selbst sein wollen." (Hirsch-Ausgabe, 8) 
2.

Wenn ich richtig sehe, ist der von Annemarie Pieper in Anschluss an Kierkegaard angeschlagene existenzphilosopische Ton zwar vielleicht noch innnerhalb des Beratungsgespräch in einer Philosophischen Praxis, aber höchstens noch am Rande in einer sich als möglichst 'wissenschaftlich $\mathrm{zu}$ profilieren bestrebenden Philosophie an der Akademie erwünscht. Das gilt besonders dort, wo sich die Philosophie in primär wissenschaftstheoretischer Absicht am Mainstream der anglo-amerikanischen analytischen Philosophie orientiert. Wahrscheinlich suggeriert der von mir - zugegebenerweise pejorativ - gebrauchte Begriff des Mainstreams eine zu grosse Einheit in dem, was gegenwärtig an Universitäten und in philosophischen Journals in hochtechnischer Weise als Philosophie betrieben wird. Es geht mir aber auch weniger darum, diese Seite, wie sman heute im Allgemeinen Philosophie versteht und betreibt, korrekt zu beschreiben - mein Interesse richtet sich vielmehr darauf, ob es doch nicht immer noch so etwas wie einen Gegen- oder Unterstrom gibt in der gegenwärtigen Philosophie, in welchem auch der existenziell an- und aufgeregte Ton eines Kierkegaards noch einen Ort fände.

3.

Jemand, der auf diesen Unterstrom in den letzten Jahren immer wieder aufmerksam gemacht hat, ist der amerikanische Philosoph Stanley Cavell. Schon in seinem frühen Aufsatz Existentialism and Analytical Philosophy (1964) hat er gezeigt, dass sich zwar nicht in der analytischen Philosophie als solcher, aber doch bei einem ihrer Gründerväter, eine Affinität zum existenzphilosophischen Denken Kierkegaards zeige. Cavell denkt dabei an den Wittgenstein der Philosophischen Untersuchungen. Es geht ihm dabei also um mehr (oder um anderes) als zu zeigen, dass Wittgenstein in seinem Verständnis des religiösen Glaubens von Kierkegaard in eminenter Weise geprägt ist. Natürlich ist dies auch der Fall und in vielen von Wittgensteins Tagebucheinträgen, besonders aber in einzelnen Passagen in den als Vermischte Bemerkungen publizierten Texten schon fast überdeutlich. Cavell interessiert sich aber dafür, inwiefern Wittgensteins Denken insgesamt eine innere Verwandschaft zu demjenigen von Kierkegaard aufweist. (Konkret bezieht er sich vor allem auf den `Kierkegaard der Unwissenschaftlichen Nachschrift und in einem anderen Text - 
Kierkegaards' on Authority and Revelation - auf denjenigen des Buches über Adler.)

Wiewohl der spätere Wittgenstein gerne in die ordinary language philosophy eingeordnet wird, macht Cavell darauf aufmerksam, dass Wittgenstein Fragen stellt und dass damit Interessen zum Vorschein kommen, die in dieserWeise bei Philosophen wie Moore oder Austin kaum zum Tragen kommen (und bei den logischen Empiristen sowieso nicht). Wittgenstein sagt im $\$ 116$ der Philosopischen Untersuchungen, es gehe darum, die Wörter »von ihrer metaphysischen, wieder auf ihre alltäglicheVerwendung« zurückzuführen. Dabei geht es ihm aber nicht nur darum aufzuzeigen, wie die Wörter in der Philosophie gleichsam den Boden unter den Füssen verlieren und im Leeren herumzuzappeln beginnen. Ihn interessiert vielmehr auch und vor allem, wieso und warum es dazu kommt, dass wir uns beim Philosophieren immer wieder ins Metaphysische versteigen und was es mit der Sprache auf sich hat, dass sie einen solchen philosophischen Eskapismus überhaupt erlaubt oder sogar motiviert. Darum schreibt Wittgenstein in den Philosophischen Untersuchungen in Form eines Selbstgesprächs, den Confessiones von Augustinus darin ähnlich, dass er sich im Modus der Selbsterkundung - aus unterschiedlichsten Perspektiven und in verschiedensten Stimmungen - selbst daraufhin erkundet, welche Versuchungen, Unzufriedenheiten und Zwänge ihn immer wieder von neuem aus dem Alltäglichen hinaus in die (auf metaphysische Weise betriebene) Philosophie hinein führen. Wenn man auf diese Weise `bekennt‘, dann erklärt oder rechtfertigt man nicht, sondern beschreibt, wie es um einen steht. (Wieso dies keinen Verzicht auf Intersubjektivität darstellt, sondern einen Vorschlag, wie man zu solcher allererst gelangen könnte, ist ein anderes Thema, auf das ich jetzt nicht eingehen möchte.)

Es ist diese Form des Selbstgesprächs, in der Cavell eine grosse Nähe zur Existenzphilosophie und deren Unterscheidung zwischen

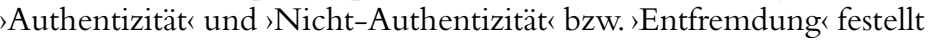
- und damit auch zur Redeweise Kierkegaards:

"The force of this mode of composition depends upon whether the interlocutors voice questions and comments which come from conviction, which are made with passion and attention, and which, as one reads, seem always something one wants oneself to say, or feels the power of. If they do, then their voices cannot, in any obvious way, be criticized or dismissed. If these voices were to be described in one word, the one that for me best captures the experiences they suggest is the existentialist's term sinauthentic, $<$ the new term of philosophical criticism directed against 
our lives. I have said that for the Oxford philosopher, ’ordinary language and >ordinary contexts 'nonphilosophical languager and snonphilosophical contexts.< In Wittgenstein's work, >ordinary examples are, I suggest, meant to carry the force of rauthentic examples authentically responded to in language. [...]

Both Wittgenstein and Kierkegaard see their worlds as laboring under illusion. Both see their function as authors to be the uncovering or diagnosing of this illusion, and freeing us from it. In both, the cure requires that we be brought (back) to our human existence." (St. Cavell, Existentialism and Analytical Philosophy, 216f).

Cavell weiss darum, dass es ein Unterschied ist, ob man die Illusion darin sieht, dass angenommen wird, dass man ein Christ einfach dadurch sei, dass man in einem christlichen Staat geboren wird einerseits oder in der Illusion, dass unsere Sprache in einer perfekten und allgemeinen Logik fundiert sei, andererseits. Und doch besteht eine grosse Ähnlichkeit darin, dass die Kur dieser Illusion bei beiden darin besteht, zu unserer gewöhnlichen Existenz zurückkehren.

4.

Statt nun noch einmal genauer auszufuihren, wieso es mir auch im Blick auf Wittgenstein legitim erscheint, die Zurückführung der Wörter "von ihrer metaphysischen [...] auf ihre alltägliche Verwendung" als eine Rückkehr von uns selbst in die alltäglichmenschliche Existenz zu beschreiben - und dabei die Ähnlichkeiten zu Kierkegaard herauszustreichen -, möchte ich in aller Kürze daran erinnern, wie sich Cavells Lesart von Wittgenstein als >existenzialistischem Sprachphilosophen in seiner Auseinandersetzung mit dem Thema des Skeptizismus ausgewirkt hat. Ich kehre damit auch langsam wieder zum Thema meines Artikels - dem Verhältnis von Zweifel und Verzweiflung - zurück. Zeigt sich bei Cavell zumindest ansatzweise, wie bei Kierkegaard dann sehr deutlich, der Versuch, den Zweifel in Richtung auf das Phänomen der Verzweiflung hin weiterzudenken?

Wir haben gesehen, wie sich der ordinary language-Philosoph (und vielleicht liesse sich Ähnliches auch im Blick auf den Pragmatismus und dessen Vertrauen in die Welt sagen) wenig dafür interessiert, wieso wir beim Philosophieren immer wieder von neuem nach dem Metaphysischen streben. Könnte sich - so fragt 
dagegen nun Cavell in Anschluss an Wittgenstein - im epistemologischen Problem des Skeptizismus nicht ein tieferes, praktischexistenzielles Problem ausdrücken, ein Problem, das wir auf philosophische Weise nicht nur nicht widerlegen können, sondern auch nicht sollen? Im Hintergrund von Cavells Überlegungen steht dabei ein recht weites Verständnis von Skeptizismus: Skeptizismus meint zum einen die Enttäuschung darüber, dass unser Wissen und Sprechen (und damit unsere Beziehung zur Welt, zu den andern und $\mathrm{zu}$ uns selbst) bloss menschlich bedingt ist und - damit eng verbunden - zum andern den Versuch, dieses bloss Gewöhnlich-Menschliche zu überwinden. Unter Skeptizismus versteht Cavell also weniger eine bestimmte Position oder philosophische Tradition als den grundmenschlichen Impuls, die menschlich-endlichen Bedingungen unseres Sprechens und Erkennens zu transzendieren. (Inwieweit dies tatsächlich ein rallgemein-menschlicher Impuls ist, muss jeder an sich selbst ausprobieren.) Wird Skeptizismus in einem solchen weiten Sinn verstanden, dann ist nicht nur derjenige ein Skeptiker, der unsere Erkenntnisfähigkeit im Blick auf die äussere Welt und den Andern radikal in Zweifel zieht, sondern es kann auch eine Form von Skeptizismus sein, was als Widerlegung des Skeptizismus (im traditionellen Sinne) intendiert ist.

Cavell spricht in diesem Zusammenhang von der Transformation des existenziellen Zweifels in ein intellektuelles Problem. Durch eine solche Transformation geht der Sinn dafür verloren, welche Erfahrungen den Philosophierenden zum Skeptizismus allererst motivieren. Im Blick auf das sogenannte other-mind-Problem beschreibt Cavell diese Erfahrung folgendermassen:

"The truth here is that we are separate, but not necessarily separated (by something); that we are, each of us, bodies, i.e., embodied; each is this one and not that, each here and not there, each now and not then. If something separates us, comes between us, that can only be a particular aspect or stance of the mind itself, a particular way in which we relate, or are related (by birth, by law, by force, in love) to one another". (St. Cavell, The Claim of Reason. Wittgenstein, Skepticism, Morality, and Tragedy, 369)

Das theoretisch-epistemologische Problem des Anderen lässt sich tatsächlich in Frage stellen, indem sinnkritisch gezeigt wird, dass es sich nicht einmal kohärent ausdrücken lässt. Es besteht aber die Gefahr, dass eine solche `Überwindung des Skeptizismus auf eine Weise geschieht, die gleichsam das Kind mit dem Bade ausschüttet - droht doch die Gefahr, dass mit dieser Überwindung die Unterdrückung 
alltäglicher Erfahrungen einhergeht. Mag das epistemologische Problem des Anderen auch ein Nicht-Problem sein - was wäre, wenn sich in ihm ein ernsthaftes, praktisch-existenzielles Problem verbirgt? Was passiert, wenn der Skeptiker und der Anti-Skeptiker - weil sie das Bedürfnis teilen, der Kontingenz und Fragilität des Gewöhnlichen zu entkommen - die menschliche Endlichkeit und die damit verbundene konstitutive Getrenntheit vom Anderen in ein intellektuelles Problem verwandeln? Nach Cavell ist ihre Sichtweise auf das Problem des Anderen zumindest einseitig. Sie verstehen es als ein Problem des Erkennens, obwohl es zumindest auch ein existenzielles oder moralisches ist: Anerkenne ich den Anspruch, der mir mit dem Anderen begegnet oder weise ich ihn zurück? Antworte ich auf das schmerzverzerrte Gesicht des Anderen mit Mitgefuhl oder mit Kälte und Indifferenz?

Zwar betont Cavell, dass der Skeptiker und der Anti-Skeptiker von demselben sskeptischen (im weiten Sinne) Impuls bestimmt sind - aber er spricht dabei immerhin auch von der /Wahrheit des Skeptizismusı. Auch wenn der Skeptiker seine Position nicht wirklich kohärent zum Ausdruck bringen kann und uns seine Worte darum nicht gänzlich verständlich sind, immerhin kommt in seinen Argumentationen eine Wahrheit unserer conditio humana zum Ausdruck, die wir nicht einfach unterdrücken, sondern mit der wir uns auseinandersetzen sollten. So sind - nach Cavell -Wittgensteins Philosophische Untersuchungen nicht als Widerlegung einer (skeptischen) These zu lesen, sondern als unabschliessbares Gespräch mit der Wahrheit des Skeptizismus: als Gespräch mit unserer skeptizistischmetaphysischen Stimme und deren Gefühl der Enttäuschung und Abgründigkeit angesichts der menschlich-gewöhnlichen Verfasstheit unseres Sprechens und Erkennens.

5.

Cavells Darstellung von Wittgensteins Auseinandersetzung mit dem Skeptizismus ist nicht die übliche. \Man kann nicht mit dem Zweifel anfangen - so habe Wittgenstein in seinem Spätwerk Über Gewissheit gezeigt. Er habe damit den epistemologischen Skeptizismus überwunden oder wenigstens zu überwinden versucht. Ungefähr dies dürfte man als Antwort bekommen, würde das philosophische Allgemeinbewusstsein daraufhin befragt, was sich ihm von Wittgensteins Auseinandersetzung mit dem philosophischen Skeptizismus eingeprägt hat. Auch innerhalb der Religions- 
philosophie und Theologie hat diese Wittgenstein-Deutung ihre Spuren hinterlassen. Nicht wenige, welche in der modernen Skepsis gegenüber dem religiösen Glauben schon lange ein blosses Vorurteil vermutet haben, begrüssen, dass sich die Begründungsanforderung nun umgekehrt zu haben scheint. Nicholas Wolterstorff hat es prägnant so ausgedrückt: Die Glaubensüberzeugungen sind »innocent until proved guilty, not guilty until proved innocent."Aber eben nicht erst diese religionsphilosophische und theologische Rezeption, bereits die Auffassung, Wittgenstein habe den Skeptizismus zu widerlegen versucht, kommt selbstverständlicher daher, als es in Tat und Wahrheit der Fall ist. Darauf hat meines Erachtens Cavell mit Recht aufmerksam gemacht.

Dabei scheint mir diese existenzielle Interpretation des Problems des Skeptizismus aber gerade auch hilfreich sein, um das Verhältnis von Zweifel und religiösem Glauben zu beschreiben. Ich zitiere eine Passage aus Kierkegaards Krankheit zum Tode:

»Ist Verzweiflung ein Vorzug oder ein Mangel? Rein dialektisch ist alles beides. Hielte man den abstrakten Gedanken der Verzweiflung fest, ohne irgendeinen Verzweifelten zu denken, müßte man sagen: sie ist ein ungeheurerVorzug. Die Möglichkeit dieser Krankheit ist des Menschen Vorzug vorm Tiere, und dieser Vorzug zeichnet ihn auf ganz andre Weise aus als der aufrechte Gang; denn sie deutet hin auf das unendliche Aufgerichtetsein oder die unendliche Erhabenheit, daß er Geist ist. [...] Es steht mit dem nicht verzweifelt Sein, nicht ebenso wie mit dem nicht lahm, nicht blind oder dergleichen Sein. Wofern das nicht verzweifelt Sein nicht mehr und nicht weniger bedeutet, als daß man es nicht ist, so heißt es gerade daß man es ist. Nicht verzweifelt sein, es muß bedeuten die zunichte gemachte Möglichkeit, es sein zu können; soll es wahr sein, daß ein Mensch nicht verzweifelt ist, so muß er jeglichen Augenblick die Möglichkeit zunichtemachen. Dergestalt ist das Verhältnis von Möglichkeit und Wirklichkeit sonst nicht." (10f in der Hirsch-Ausgabe)

Ronald Hall spricht in seinem Buch The Human Embrace von Kierkegaards `Logik des Paradoxes im Rahmen seiner recht eigenwilligen Deutung des ästhetischen, des ethischen und des religiösen (Religiosität A) Stadiums auf der einen und des existenziellen Glaubens (Religiosität B) auf der anderen Seite: Die drei Alternativen zum existenziellen Glauben sind alle durch das Strukturelement der Resignation und Zurückweisung bestimmt. Nur der existenzielle Glaube vollzieht sich als wahre und 
volle Bejahung unseres menschlichen Daseins innerhalb der endlichen geschichtlichen Welt, aber doch zugleich so, dass die Resignation und die Zurückweisung auch zu Strukturelementen innerhalb des Glaubens werden. Dies ist darum so, weil der existenzielle Glaube unmöglich wäre, wenn die Resignation und die Zurückweisung nicht wirkliche, wenn auch ausgeschlossene und immer wieder von neuen auszuschliessende, existenzielle Möglichkeiten wären. Dem Zweifel, dem Wissen um die mögliche Enttäuschung und die Ungesichertheit unserer Beziehung zum anderen, kommt die positive Funktion zu, (neue Möglichkeiten erschliessend) auf andere und intensivere Formen der Teilhabe an unserem gewöhnlichen Leben aufmerksam zu machen.

6.

Allerdings - darauf hat mich Johannes Corrodi (von ihm stammt ebenfalls ein Text im vorliegenden Band) vor einiger Zeit aufmerksam gemacht -, wie gehe ich damit um, dass Kierkegaard an dieser Stelle in Die Krankheit zum Tode gar nicht von Zweifel oder Skeptizismus spricht, sondern von Verzweiflung? Ich habe auf diese Frage keine klare Antwort. Aber ich möchte zum Schluss wenigstens andeuten, in welche Richtung ich mich dabei auf die Suche mache.

$\mathrm{Zu}$ klären wäre zuerst einmal, was Kierkegaard unterVerzweiflung versteht und wie er ihn vom Zweifel unterscheidet. Dazu wäre ein Durchgang durch Kierkegaards ganzes Werk nötig, wie es zum Beispiel Kristin Kaufmann in ihrer Dissertation Vom Zweifel zur Verzweiflung. Grundbegriffe der Existenzphilosophie Sören Kierkegaards getan hat. Kaufmann hat Kierkegaards Schriften insgesamt als Auseindersetzung mit dem Zweifel interpretiert und gezeigt, dass der Weg, den Kierkegaard gegangen ist, schon sehr früh in den sogenannten Briefen eines jungen faustischen Zweiflers angelegt ist:Während der methodische Zweifel Descartes oder der ssich selbst vollbringende Skeptizismus< Hegels aus sich heraus zu einer neuen Gewissheit führt, mündet der radikale, faustische Zweifel in einen existenziellen Zweifel - in die Verzweiflung. Das spätere Werk Kierkegaards lässt sich demnach als Auseinandersetzung und als Antwortversuch auf diesen, als Verzweiflung verstandenen, existenziellen Zweifel lesen. Ich muss mich an dieser Stelle auf den Hinweis auf eine exemplarische Passage aus Kierkegaards Entweder/Oder, wo dieser Unterschied zwischen dem epistemologisch-theoretischen und dem existenziellpraktischen Zweifel besonders prägnant zum Ausdruck kommt, 
beschränken (auf diesen Text bin ich dank Stephen N. Dunning, The Illussory Grandeur of Doubt gestossen):

"Es ist in der neueren Philosophie überreichlich davon die Rede gewesen, daß alle Spekulation anhebt mit dem Zweifel; dahingegen hab ich, soweit ich mich gelegentlich mit solchen Erwägungen habe beschäftigen können, vergeblich eine Aufklärung gesucht, worin denn Zweifel von Verzweiflung unterschieden sei. [...] Zweifel ist des Gedankens Verzweiflung, Verzweiflung ist der Persönlichkeit Zweifel, das ist der Grund, weshalb ich so festhalte an der Bestimmung des Wählens, welche meine Losung ist, der Nerv meiner Lebensanschauung, und eine solche hab ich, wiewohl ich mir keineswegs anmaße, ein System $\mathrm{zu}$ haben. Zweifel ist die innere Bewegung des Gedankens selber, und in meinem Zweifel verhalte ich mich so unpersönlich als möglich. Ich will nun annehmen, daß der Gedanke, indem der Zweifel durchgeführt wird, das Absolute finde und darin ruhe; alsdann ruht er darin nicht einer Wahl zufolge, sondern der gleichen Notwendigkeit zufolge, gemäß deren er gezweifelt hat; denn der Zweifel selber ist eine Bestimmung von Notwendigkeit und das Ruhen desgleichen. Dies ist das Erhabene am Zweifel, um dessentwillen er von Leuten, die schwerlich verstanden, was sie sagten, so oft angepriesen und ausgeschrieen worden ist. Der Umstand aber, daß es eine Bestimmung der Notwendigkeit ist, beweist, daß die ganze Persönlichkeit nicht mit in Bewegung ist." (Entweder/Oder, 2. Teil, 224f)

"Zweifel ist des Gedankens Verzweiflung, Verzweiflung ist der Persönlichkeit Zweifel « - das ist zwar nicht die Antwort, welche der Richter William auf den faustischen Zweifel des Pseudonym A gibt. William widerspricht dem (ästhetischen) Zynismus von A, indem er an diesen appelliert, sich (ethisch) als die Person zu wählen, die er ist, auch wenn dies bedeuten würde, die eigene Verzweiflung zu wählen. In diesem Zusammenhang unterscheidet er aber zwischen dem Zweifel der modernen Philosophie, die alle ihre Spekulation mit dem Zweifel beginnen will, einerseits und der Erfahrung der Verzweiflung andererseits - und im Lichte dieser Unterscheidung denkt Kierkegaard (mithilfe seiner verschiedenen Pseudonyme) dann weiter. Der grundlegende Unterschied zwischen Zweifel und Verzweiflung besteht darin, dass der Zweifel unpersönlich, die Verzweiflung aber etwas zutiefst Persönliches ist. Der Zweifel ist einzig ein Geschäft des Intellekts, die Verzweiflung involviert die ganze Persönlichkeit. Verzweiflung ist darum auch nicht nur Selbst- 
Zweifel in dem Sinne, dass nun einfach - in demselben (möglichst) unpersönlichen Sinne - das Selbst Gegenstand des Zweifels würde. Um es nochmals mit Annemarie Pieper zu sagen: „Verzweiflung als existenzielle Befindlichkeit ist radikalisierter Selbstzweifel, der den ganzen Menschen, nicht nur den Kopf erfasst."

Selbstzweifel in diesem existenziellen Sinne kann also nicht persönlich genug sein! War das aber nicht der Weg, den auch Cavell, in Anschluss an Wittgenstein, der Philosophie zu gehen vorgeschlagen hat? Einiges spricht meines Erachtens dafür, dass dort, wo Cavell in existenziell-praktischer Weise vom Zweifel spricht, die Sphäre dessen, was Kierkegaard mit Verzweiflung meint, zumindest berührt. Es wäre dies schliesslich ein interessanter Punkt, um nachzufragen, inwiefern man darum nicht nur bei Kierkegaard, sondern auch bei Cavell auf das Thema der Sünde zu sprechen kommen müsste, wollte man verstehen, worum es sich beim radikalem Selbst-Zweifel eigentlich handelt.

- Dr. Andreas Hunziker ist geschäftsführender Oberassistent am Institut für Hermeneutik und Religionsphilosophie an der Universität Zürich. 